\title{
The ternary quantum-dot cell and ternary logic
}

\section{Lebar Bajec, N Zimic and M Mraz}

University of Ljubljana, Faculty of Computer and Information Science, 1000 Ljubljana, Slovenia

E-mail: iztok.bajec@fri.uni-lj.si

\begin{abstract}
Quantum-Dot Cellular Automata (QCAs) are becoming more and more one of the most promising candidates for the alternative processing platform of the future. Since their advent in the early 1990s the required technological processes, as well as the QCA structures that implement the basic and functionally complete set of binary logic functions, have been developed. This article, however, presents an extension of the (standard) binary quantum-dot cell that is focused on the enrichment of the cell's processing capabilities. It is shown that the newly introduced ternary quantum-dot cell can be used to represent three logic values and that only minor modifications of the corresponding binary QCA structures are required to implement the functionally complete set of Eukasiewicz ternary logic functions.
\end{abstract}

Submitted to: Nanotechnology 


\section{Introduction}

With the constant miniaturisation of logic gates and the corresponding processing structures, which as evident in the last decade strictly follows Gordon Moore's law, it is to be expected that the first nano-scale $\left(10^{-9}\right)$ processing entities (e.g. logic gates) will appear in the decade to come [1]. The above mentioned scale corresponds to the size of molecules and atoms, which means that this is what will actually have to be mastered through physical and chemical theory and technological processes. It also means that upon this point the eventual further miniaturisation will be drastically slowed down. Due to this there already exists quite a lively search for the alternative processing platform of the future. It can be noticed in fields such as 'DNA', 'bimolecular', 'quantum', 'quantum cellular', etc. computing. The alternative processing platform of the future should, however, meet the following criteria:

- from the point of view of reliability and performance it has to allow for a transparent transition from the transistor based platform,

- from the point of view of energy consumption it has to be economically viable,

- it has to allow for future improvements in processing capabilities, and

- processing on this platform, due to its eventual energetic self-sufficiency, should never get out of control [2].

The criterion that proves to be the most difficult one to meet is the one requiring reliability and performance that is similar to those obtainable, using the present transistor based platforms. One of the few platforms, which according to the existing publications, meets this criterion as well as all of the rest, is the Quantum-Dot Cellular Automaton, or QCA. It was set up by C.S. Lent [3, 4] at the start of the last decade as a planar structure comprised of cells, each containing a set of four quantum-dots separated by tunnelling barriers and occupied by two electrons. According to Lent, each cell can be in one of two distinct states, distinguishable by means of the arrangement of the electrons. The growing interest in QCAs in the last decade resulted in the development of QCA structures that implement the functionally complete set of binary logic functions $\{\mathrm{AND}, \mathrm{OR}, \mathrm{NOT}\}[3,4,5]$, as well as the development of tools that enable the design and simulation of more complex QCA structures [6].

The primary drawback of QCAs, however, is ironically the bi-stability of the comprising cells. In fact, most of the research in QCAs is dedicated to the implementation of the classical binary logic and the corresponding computer structures. But the use of binary logic in computer structures was historically necessary only due to technological limitations, which means that the alternative processing platforms of the future should by no means disregard the possibility of implementing and using multivalued logic. The benefits of using multi-valued processing are obvious and range from higher data storage capabilities to faster and more sophisticated processing [7]. This is why we, with the premise that there are no technological limitations and that more than four quantum dots can be arranged on a cell's surface, devoted our research to the 
development of a quantum-dot cell that would be capable of multi-valued processing. When used for the construction of QCA structures the inter-cellular distance will be taken to be the same as in Lent's case. This will, assuming that the use of the new cell is transparent (i.e. when employing it to construct QCA structures used to implement the binary logic functions the latter operate in a multi-valued sense), give the new cell an advantage of $m / 2$ over Lent's cell, where $m$ is the number of values the new cell is capable of representing.

This article, therefore, presents our first step in that direction - it presents an extension of the standard QCA cell. The principal advantage of the newly introduced cell is its capability of ternary processing. We begin the article with a condensed review of the binary quantum-dot cell and the associated binary QCA structures. We continue with the introduction of the ternary quantum-dot cell and in the rest of the paper demonstrate that while using it, all of the existing solutions for transmitting data still work, this time however in the sense of ternary logic. What is more, we demonstrate that with the ternary quantum-dot cell we are capable of implementing the functionally complete set of ternary logic functions originally introduced by J. Łukasiewicz [8].

\section{The QCA cell}

The standard QCA cell, or binary quantum-dot cell, typically comprises four quantum dots arranged in a square formation $[9,10,11]$. The near-neighbour distance between dot centres $a$ is usually taken to be $20 \mathrm{~nm}$. The cell is occupied by two electrons, which can tunnel only between adjacent dots, whereas their tunnelling out of the cell is supposed to be completely suppressed. The tunnelling occurs due to Coulomb interaction between the electrons themselves, or between them and the environment. Due to the associated repulsive force the electrons tunnel into the dots with the energetically favourable positions. Within a single cell the electrons tend to align along one of the two diagonals, as these correspond to arrangements with their maximum spatial separation. In the absence of external electric fields these two arrangements have exactly the same energy, however in the presence of an external electric field one of them is energetically favoured. This provides the QCA cell with the so desirable bi-stability.

The architecture of the four-dot two-electron binary quantum-dot cell is presented in figure 1(a). Figure 1(b), on the other hand, presents the two diagonal arrangements, as well as the four additional possible electron arrangements. $\ddagger$ The arrangements with more than one electron occupying a quantum dot are not considered owing to the extremely large associated repulsive force. The two diagonal arrangements are due to their bistability interpreted as logic values 0 and 1 , whereas the rest of the arrangements are due to the large associated repulsive forces interpreted as ' $\mathrm{X}$ ' or unfavourable states. In other words, the cell's state is, from the point of view of processing, denoted by the arrangement of the contained electrons.

$\ddagger$ Note that for quantum-dot cells in all but figures $1(a)$ and $4(a)$ only the electron arrangement is displayed. 
(a)

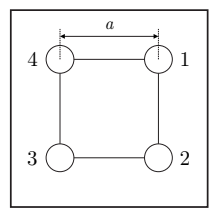

(b)
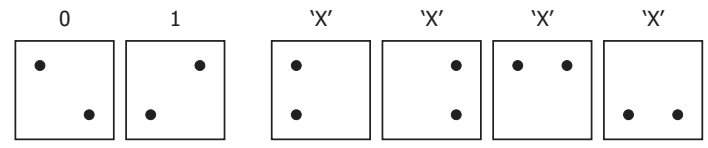

Figure 1. A QCA cell with four quantum dots and the corresponding tunnelling paths (a) and the possible arrangements of the two occupying electrons $(b)$.

\subsection{The QCA structures}

As already discussed, Lent et al. interpret a two-dimensional arrangement of quantumdot cells as a QCA $[3,4]$. In a QCA the near-neighbour distance between cell centres is typically $3 a$. In addition, a QCA designed to perform a specific task is usually referred to as a QCA structure. The synchronisation of information flow within a QCA structure is obtained by means of 'allowed' tunnelling — by applying the corresponding time sequence of cell 'locking' that disallows the tunnelling of electrons. The time sequence is not applied to the processing units (cells/structures) directly, but instead it is applied in the form of an electric field, whose (presence)absence (dis)allows tunnelling within the designated processing unit. A more detailed description of the locking mechanism, which involves four phases named switch, hold, release and relaxed, and is used in clocked QCA architectures, can be found in $[12,13]$. This paper, however, restricts to unclocked QCA architectures. Regardless of the architecture used, within a QCA structure the comprising cells are not treated as equal and for this reason they are denoted using three distinct names based on the tasks they perform:

- driver cells are typically situated at the borders of the structure and their state is fixed by means of external electrostatic fields (in figures 2, 3, 5, 6 and 9 depicted by using a bold outline),

- internal cells are typically situated within the structure and are those where the actual processing and desired data 'transformation' takes place (in figures 2, 3, 5, 6 and 9 depicted in grey colour),

- target cells are again typically situated at the borders of the structure and their state (after a certain time delay) is interpreted as the processing output (in figures 2 , $3,5,6$ and 9 depicted with an ordinary outline).

\subsection{The ground state}

Within a single quantum-dot cell, when no external electric field is present, the two diagonal arrangements have exactly the same energy. This means that a cell by itself is overall neutral. From the modelling point of view this is achieved by assigning to each 
quantum dot a positive charge of $\left(n e_{0}\right) / m$, where $n$ is the number of electrons, $e_{0}$ the electron charge and $m$ the number of dots (e.g. in the case of the binary quantum-dot cell $n=2$ and $m=4)$. An electron occupied dot thus has a charge of $\left(n e_{0}\right) / m-e_{0}$, which means that the sum of charges within a single cell equals 0 , and that the energy associated with the two diagonal arrangements is exactly the same. Upon the premise that there are two cells, an energetically neutral cell circumvents the problem where the electrons in the target cell are pushed away from the driver cell (i.e. tunnel into dots that are further away from the driver cell). The described effect is associated with the monopole electric field component that would be produced by a non-neutral cell [11].

A QCA structure can be observed from the point of view of total electrostatic energy [11], which is expressed as the energy of a system of point charges

$$
E=\sum_{i \neq j} \frac{\varrho_{i} \varrho_{j}}{4 \pi \varepsilon_{0} \varepsilon_{r} r_{i j}}
$$

where $\varrho_{i}, \varrho_{j}$ are the charges associated with dots $i$ and $j, r_{i j}$ is their distance, $\varepsilon_{0}$ is the vacuum permittivity, and $\varepsilon_{r}$ is the relative permittivity of the medium (our model assumes the medium is GaAs/AlGaAs, whose relative permittivity is roughly 12.9). By repeating the evaluation of the total electrostatic energy for all combinations of electron arrangements the energetically minimal one, or ground state, can be found. Nevertheless, such an exhaustive exploration does not come cheap as for a QCA structure constructed of $k$ quantum-dot cells, each comprised of $m$ quantum dots and occupied by $n$ electrons, this means $\left(\begin{array}{c}m \\ n\end{array}\right)^{k}$ evaluations. The described method, where the electrons are treated as classical particles, which can however tunnel between adjecent quantum dots, is also known as the semi-classical model [11].

\section{Binary processing using binary QCA structures}

In the 1990s a group of researchers led by Lent $[3,4,5]$ succeeded in designing a series of QCA structures, whose dynamics corresponds to the functionally complete set of binary logic functions $\{$ AND, OR, NOT\} [14]. Their solution is, besides being interesting from the point of view of the possibility to design any desirable binary logic function, interesting also due to the way by which the binary AND and OR logic functions are implemented. Indeed, these are implemented by using the so called majority gate, which due to its generality gives reason to believe there is a possibility of designing more efficient processing structures than the currently available transistor based ones.

The implementation of the binary NOT logic function is presented in figure $2(a)$, whereas figure $2(b)$ presents the implementation of the binary AND and OR logic functions. In the latter case the cells marked as $X_{1}$ and $X_{2}$ are driver cells by means of which the values of the input variables enter the structure. The cell marked as $S$ is, on the other hand, the driver cell by means of which the designer selects the behaviour of the structure (i.e. as the binary OR logic function when it is set to the logic value 1 , and as the binary AND logic function when it is set to the logic value 0 ). In addition to the functionally complete set of binary logic functions the researchers presented a special 
(a)

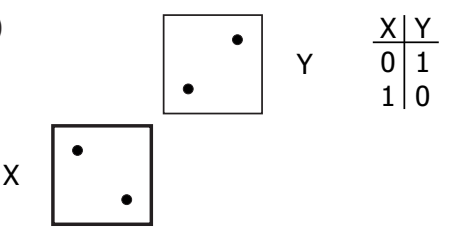

(b)

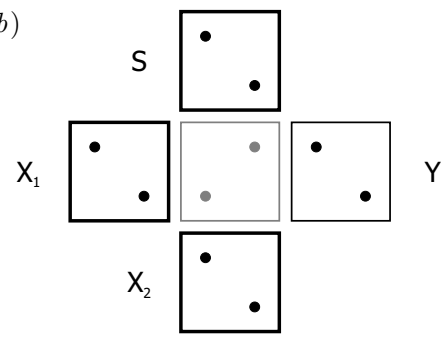

\begin{tabular}{lll|l}
$S$ & $X_{1}$ & $X_{2}$ & $Y$ \\
\hline 0 & 0 & 0 & 0
\end{tabular}

永

$0 \begin{array}{lllllll}0 & 1 & 0 & 0\end{array}$

$<\begin{array}{llll}0 & 1 & 1 & 1\end{array}$

\begin{tabular}{lll|l}
1 & 0 & 0 & 0
\end{tabular}

\begin{tabular}{ccc|ccc}
$\times$ & 1 & 0 & 1 & 1 \\
\hdashline & -1 & 1
\end{tabular}

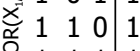

$\begin{array}{llllllll}1 & 1 & 1 & 1\end{array}$

Figure 2. Implementation of the binary logic functions NOT (a), AND and OR (b).

structure that is capable of transmitting binary data and named it the binary wire (see figure 3).

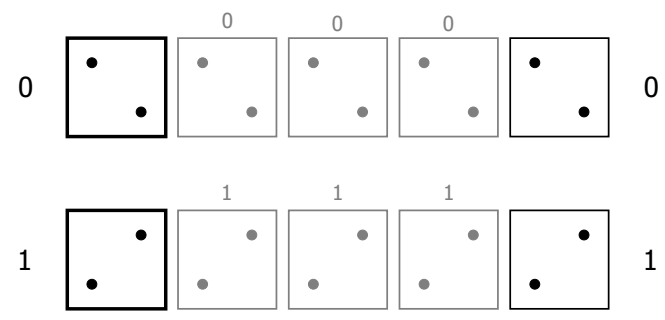

Figure 3. Transfer of a binary logic value along a wire of binary quantum-dot cells.

The basic characteristics of computer systems are processing, data transfer and data storage. As already discussed, the QCA structures are capable of both processing and data transfer, but what about data storage? In their seminal paper, Lent et al. [3] stated that a single quantum-dot cell can act as a data storage cell, since a cell, whose electrons have stabilised in a certain arrangement, will in principle remain in that configuration indefinitely. The latter can be interpreted as data preservation. In this view the QCA structures, at least in theory, possess all the necessary capabilities for implementing any given computer system [14]. If truth be told later studies showed that in the described case the cell does not preserve any data, as it becomes 'unpolarized' and thus preserves nothing, neither the logic value 0 , nor 1 . However, with the introduction of clocked QCA architectures researchers developed QCA structures that are capable of data storage $[6,15]$. This is why clocked architectures seem to currently be the preferred line of research. Nevertheless, since even the QCA structures capable of data storage are constructed using the basic QCA structures (i.e. logic functions, wires), we opted to address only the latter. Similarly we decided to address only unclocked 
architectures, as clocking, besides providing control over electron transfers within the cells, allows avoiding undesirable metastable states $\oint$ and if added would prove only beneficial. However, if an unclocked architecture proved to be sufficient, the additional reduction in complexity due to the unclocked architecture would be more than welcome fabrication wise. Research on clocked ternary architectures and QCA structures capable of ternary data storage is nevertheless one of our future lines of work.

\section{The ternary quantum-dot cell}

In the previous sections we presented a condensed overview of the binary quantum-dot cell. In view of the alternative processing platforms of the future the primary drawback in its usability is ironically its bi-stability. Based on the premise that there are no technological limitations and that there can be more than four quantum dots arranged on a cell's surface, our research focused on the study of the dynamics of a multi-state cell. In this view a quantum-dot cell comprised of eight quantum dots arranged in a circular formation and occupied by two electrons was defined [16] and denoted as the ternary quantum-dot cell. The radius of the formation $r$ was taken to be $a \sqrt{2} / 2$ so that dots 1-4 correspond perfectly with those present in the binary quantum-dot cell and that the near-neighbour distance between cell centres in a QCA structure constructed from ternary quantum-dot cells could be kept $3 a$.

The cell's architecture is presented in figure $4(a)$, whereas figure $4(b)$ presents the four electron arrangements (marked as states 'A', 'B', 'C' and 'D') that correspond to their maximum spatial separation, as well as the rest of all possible arrangements. As the latter, however, correspond to energetically unfavoured arrangements they are marked as ' $\mathrm{X}$ ' states. States 'A' and 'B' (i.e. the two diagonal arrangements) are, as in the case of the binary quantum-dot cell, interpreted as logic values 0 and 1 . In other words, there are two newly introduced states ' $C$ ' and ' $D$ ', but for reasons that will be made evident in the following sections both are interpreted as the logic value $\frac{1}{2}$ and in addition only state ' $\mathrm{C}$ ' is allowed for driver and target cells.

It is important to note that from the modelling point of view the properties of the ternary quantum-dot cell and the dynamics of the associated QCA structures are not altered in any way. In other words, all properties described in sections 2.1 and 2.2 still hold. For reasons of clarity, however, a QCA structure comprising ternary quantum-dot cells shall be denoted a ternary QCA structure and a QCA structure comprising binary quantum-dot cells a binary QCA structure.

\section{Ternary processing using ternary QCA structures}

Our research was led by the assumption that the QCA structures used to implement the binary logic functions and wire could be, by constructing them with ternary quantum-

$\S$ States that lead to a "thermal death" (unpredictable output or very long settling times) in large arrays of unclocked binary quantum-dot cells. 
(a)

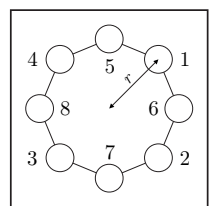

(b)
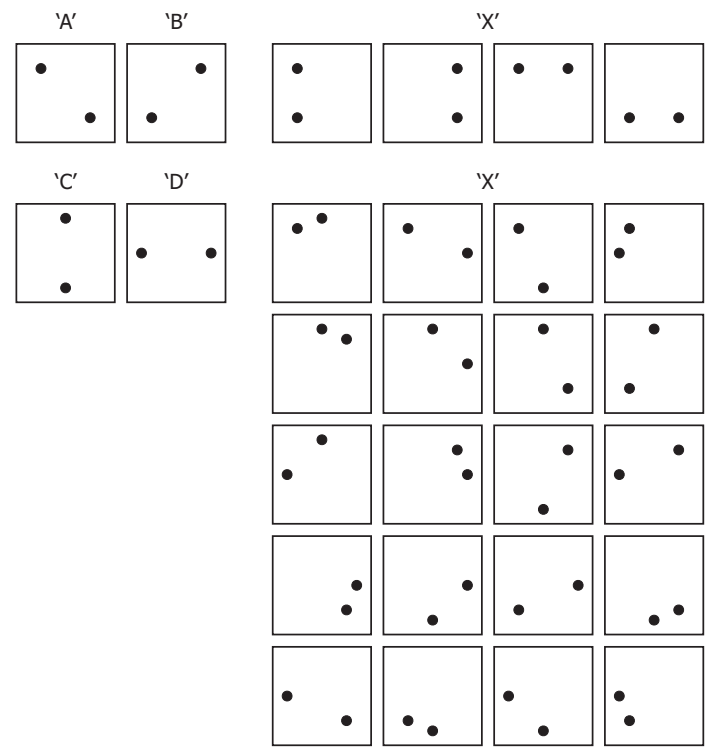

Figure 4. A QCA cell with eight quantum dots and the corresponding tunnelling paths $(a)$ and the possible arrangements of the two occupying electrons $(b)$.

dot cells, used to implement multi-valued logic functions and wire. Thus we constructed them with ternary quantum-dot cells and for each of the allowed input states computed the corresponding ground state by means of the exhaustive exploration that is explained in section 2.2. Figure 5 presents the behaviour of a wire of ternary quantum-dot cells.

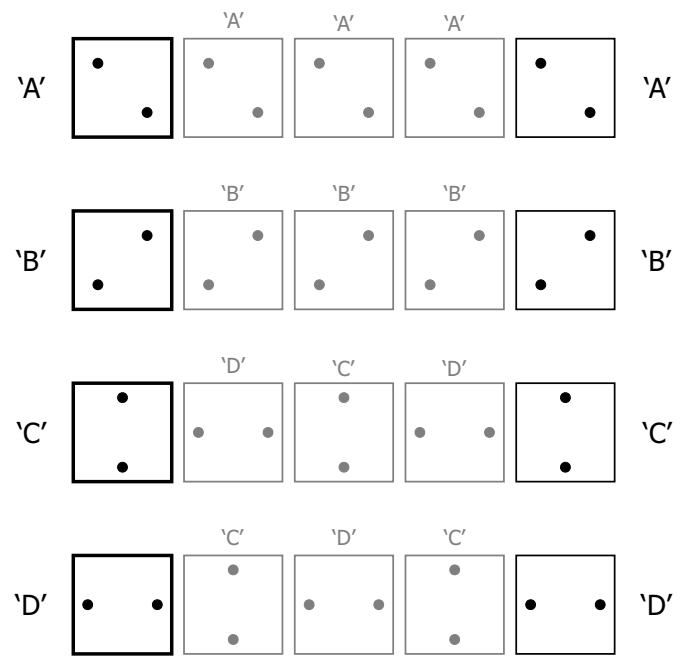

Figure 5. Behaviour of a wire of ternary quantum-dot cells. 
As said, the structure now has four possible input states. It can be noticed that when the driver cell's state is 'A' or 'B' all of the internal cells, as well as the target cell, assume the same state, which corresponds perfectly with the behaviour observed in the case of the binary wire (compare figures 3 and 5). Computing the ground state for the case when the driver cell's state is ' $C$ ' or ' $D$ ', however, showed that the internal and target cells assume alternating states. This effectively meant that either wires have to be limited to odd lengths or the two states have to be interpreted as the same logic value. For reasons of generality the latter approach was taken, which resulted in the wire's capability of transmitting three logic values 0,1 , and $\frac{1}{2}$, hance behaving as a ternary wire.

By committing to three logic values the next logic step was to see if the QCA structures used to implement the binary logic functions AND, OR and NOT, when constructed with ternary quantum-dot cells, implement their ternary counterparts. The analysis was based on the comparison with the ternary truth tables introduced by Łukasiewicz [8]. As evident from figure 6, the QCA inverter constructed by using ternary

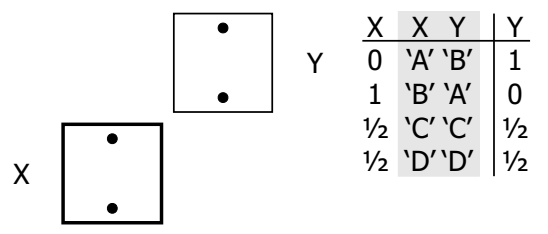

Figure 6. Behaviour of the QCA inverter when constructed by using ternary quantumdot cells.

quantum-dot cells implements the ternary NOT logic function. Indeed if the driver cell's state is ' $\mathrm{A}$ ' (logic value 0 ) the target cell assumes state ' $\mathrm{B}$ ' (logic value 1 ) and vice versa. On the other hand, if the driver cell's state is either ' $\mathrm{C}$ ' or ' $\mathrm{D}$ ' (logic value $\frac{1}{2}$ ), the target cell also assumes state ' $\mathrm{C}$ ' or ' $\mathrm{D}$ ' (logic value $\frac{1}{2}$ ), thus retaining both the driver cell's logic value, as well as state.

In the case of the majority gate structure computing the ground states for all possible input states, however, showed that the structure does not behave as intended (see figure 7). This negated our initial assumption of an easy transition to ternary logic.

\subsection{Implementation of the ternary AND and OR logic functions}

As discussed in the previous section, simulations showed that with the existing preconditions and interpretation of electron arrangements it is not possible to implement the ternary AND and OR logic functions as majority gates. Nevertheless, after a further review of the structure's behaviour it became evident that if state ' $D$ ' is treated as an internal state (i.e. is allowed only for internal cells) $\|$, a behaviour remarkably similar to

\| This precondition, however, has an after-effect on ternary wires also, as it indirectly states that only wires of odd lengths (in which case the target cell assumes the same state as the driver cell) can be constructed. 


\begin{tabular}{|c|c|c|c|c|c|c|}
\hline $\mathrm{S} \mathrm{X}_{1} \mathrm{X}_{2}$ & $Y$ & $S \mathrm{X}_{1} \mathrm{X}_{2}$ & & $\mathrm{~S} \mathrm{X}_{1} \mathrm{X}_{2}$ & & \begin{tabular}{l|l|l}
$S X_{1} X_{2}$ & $Y$ \\
\end{tabular} \\
\hline $\bar{A}^{\prime}{ }^{\prime} A^{\prime \prime} A^{\prime}$ & $\mathrm{A}^{\prime}$ & $B^{\prime \prime} A^{\prime \prime} A^{\prime}{ }^{\prime}$ & $\mathrm{A}^{\prime}$ & ${ }^{\circ} C^{\prime} A^{\prime \prime} A^{\prime}$ & $\mathrm{A}^{\prime}$ & $\bar{D}^{\prime \prime} A^{\prime \prime} A^{\prime} \mid D$ \\
\hline ' $A^{\prime} A^{\prime \prime} B^{\prime}$ & 'A' & ${ }^{\prime} B^{\prime \prime} A^{\prime \prime} B^{\prime} \mid[$ & 'D' & $\mathrm{C}^{\prime \prime} \mathrm{A}^{\prime} \mathrm{B}^{\prime}$ & ${ }^{\prime} C^{\prime}$ & $\mathrm{D}^{\prime \prime} \mathrm{A}^{\prime \prime} \mathrm{B}^{\prime} \mid \mathrm{D}^{\prime}$ \\
\hline$A^{\prime} A^{\prime \prime} C^{\prime}$ & 'A' & $\left.\mathrm{B}^{\prime} \mathrm{B}^{\prime \prime} \mathrm{A}^{\prime}\right|^{\prime}$ & ${ }^{\prime} C^{\prime}$ & ${ }^{\prime} C^{\prime} A^{\prime \prime} C^{\prime} \mid '$ & ${ }^{\prime} C^{\prime}$ & $D^{\prime \prime} A^{\prime \prime} C^{\prime} \mid A$ \\
\hline$A^{\prime} A^{\prime \prime} D^{\prime}$ & 'D' & ${ }^{\prime} B^{\prime} A^{\prime \prime} D^{\prime} \mid '$ & 'D' & ${ }^{\prime} C^{\prime} A^{\prime \prime} D^{\prime}$ & 'A' & ' $D^{\prime \prime} A^{\prime \prime} D^{\prime} \mid ' D$ \\
\hline${ }^{\prime} A^{\prime} B^{\prime \prime} A^{\prime}$ & 'D' & $\mathrm{B}^{\prime} \mathrm{B}^{\prime} \mathrm{A}^{\prime}$ & 'B' & $\mathrm{C}^{\prime} \mathrm{B}^{\prime} \mathrm{A}^{\prime}$ & ${ }^{\prime} C^{\prime}$ & $D^{\prime} D^{\prime \prime} B^{\prime} A^{\prime} \mid ' D$ \\
\hline$A^{\prime} B^{\prime \prime} B^{\prime}$ & 'B' & ${ }^{\prime} B^{\prime} B^{\prime \prime} B^{\prime} \mid '$ & 'B' & ${ }^{\prime} C^{\prime} B^{\prime \prime} B^{\prime}$ & 'B' & ${ }^{\prime} D^{\prime \prime} B^{\prime \prime} B^{\prime} \mid ' D$ \\
\hline$A^{\prime} B^{\prime \prime} C^{\prime}$ & 'C' & 'B' $\left.B^{\prime} B^{\prime \prime} C^{\prime}\right|^{\prime}$ & 'B' & ${ }^{\prime} C^{\prime} B^{\prime \prime} C^{\prime}$ & ${ }^{\prime} C^{\prime}$ & ' $\left.D^{\prime \prime} B^{\prime \prime} C^{\prime}\right|^{\prime} B$ \\
\hline$A^{\prime} B^{\prime \prime} D^{\prime}$ & 'D' & 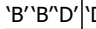 & 'D' & ${ }^{\prime} C^{\prime} B^{\prime \prime} D^{\prime}$ & 'B' & ${ }^{\prime} D^{\prime \prime} B^{\prime \prime} D^{\prime} \mid ' D$ \\
\hline$A^{\prime \prime} C^{\prime} A^{\prime}$ & 'A' & $\mathrm{B}^{\prime \prime} \mathrm{C}^{\prime \prime} \mathrm{A}^{\prime} \mid{ }^{\prime}$ & ${ }^{\prime} C^{\prime}$ & $\mathrm{C}^{\prime \prime} \mathrm{C}^{\prime \prime} \mathrm{A}^{\prime}$ & ${ }^{\prime} C^{\prime}$ & $D^{\prime \prime} C^{\prime \prime} A^{\prime} \cdot{ }^{\prime} D$ \\
\hline$A^{\prime \prime} C^{\prime} B^{\prime}$ & 'C' & $\mathrm{B}^{\prime} \mathrm{B}^{\prime \prime} \mathrm{B}^{\prime} \mid \cdot$ & 'B' & ${ }^{\prime} C^{\prime} C^{\prime \prime} B^{\prime} \mid '$ & ${ }^{\prime} C^{\prime}$ & ' $D^{\prime \prime} C^{\prime \prime} B^{\prime} \mid ' D$ \\
\hline$A^{\prime \prime} C^{\prime \prime} C^{\prime}$ & 'C' & $\left.\mathrm{B}^{\prime} \mathrm{C}^{\prime \prime} \mathrm{C}^{\prime}\right|^{\prime}$ & 'C' & ${ }^{\prime} C^{\prime \prime} C^{\prime \prime} C^{\prime}$ & ${ }^{\prime} C^{\prime}$ & $\mathrm{D}^{\prime \prime} \mathrm{C}^{\prime \prime} \mathrm{C}^{\prime} \mid \mathrm{C}^{\prime}$ \\
\hline${ }^{\prime} A^{\prime \prime} C^{\prime \prime} D^{\prime}$ & 'D' & ${ }^{\prime} \mathrm{B}^{\prime \prime} \mathrm{C}^{\prime \prime} \mathrm{D}^{\prime} \mid \mathrm{L}$ & 'D' & ${ }^{\prime} C^{\prime \prime} C^{\prime} D^{\prime}$ & ${ }^{\prime} C^{\prime}$ & ${ }^{\prime} D^{\prime \prime} C^{\prime \prime} D^{\prime} \mid D$ \\
\hline$A^{\prime \prime} D^{\prime \prime} A^{\prime}$ & 'D' & 'B' $B^{\prime \prime} D^{\prime \prime} A^{\prime} \mid '$ & 'D' & $C^{\prime} D^{\prime \prime} A^{\prime}{ }^{\prime}$ & 'A' & 'D'D' $D^{\prime \prime} A^{\prime}$ \\
\hline${ }^{\prime} A^{\prime \prime} D^{\prime \prime} B^{\prime}$ & 'D' & $\mathrm{B}^{\prime} \mathrm{B}^{\prime \prime} \mathrm{B}^{\prime} \mid \cdot$ & 'D' & $C^{\prime \prime} D^{\prime \prime} B^{\prime}$ & 'B' & 'D”D $D^{\prime \prime} B^{\prime}$ \\
\hline$A^{\prime \prime} D^{\prime \prime} C^{\prime}$ & $A^{\prime}$ & $\left.\mathrm{B}^{\prime \prime} \mathrm{D}^{\prime \prime} \mathrm{C}^{\prime}\right|^{\prime}$ & 'B' & ${ }^{\prime} C^{\prime \prime} D^{\prime \prime} C^{\prime} \mid '$ & ${ }^{\prime} C^{\prime}$ & $D^{\prime \prime} D^{\prime \prime} C^{\prime} \mid ' D$ \\
\hline & 'D' & 'B'D $D^{\prime \prime} D^{\prime} \mid '$ & 'D' & ${ }^{\prime} C^{\prime} D^{\prime \prime} D^{\prime} \mid '$ & 'D' & 'D”D $D^{n}$ \\
\hline
\end{tabular}

Figure 7. Behaviour of the majority gate when constructed with ternary quantum-dot cells.

the desired can be obtained (see shaded area in figure 7). Indeed, as presented in figure 8, in the case when the state of the driver cell marked as $S$ is ' $A$ ' the behaviour is similar

\begin{tabular}{|c|c|c|c|}
\hline & $S X_{1} X_{2}$ & $S X_{1} X_{2} Y$ & Y \\
\hline & $\begin{array}{lll}0 & 0 & 0\end{array}$ & ${ }^{\prime} \mathrm{A}^{\prime} \mathrm{A}^{\prime \prime} \mathrm{A}^{\prime} \mathrm{A}^{\prime}$ & 0 \\
\hline & $\begin{array}{lll}0 & 0 & 1 / 2\end{array}$ & $A^{\prime} A^{\prime \prime} C^{\prime} A^{\prime}$ & 0 \\
\hline & $\begin{array}{lll}0 & 0 & 1\end{array}$ & $A^{\prime} A^{\prime \prime} B^{\prime \prime} A^{\prime}$ & 0 \\
\hline & $01 / 20$ & $A^{\prime \prime} C^{\prime \prime} A^{\prime \prime} A^{\prime}$ & 0 \\
\hline & $01 / 21 / 2$ & $A^{\prime \prime} C^{\prime \prime} C^{\prime \prime} C^{\prime}$ & $1 / 2$ \\
\hline & $0 \quad 1 / 21$ & $A^{\prime \prime} C^{\prime \prime} B^{\prime \prime} C^{\prime}$ & $1 / 2$ \\
\hline & $\begin{array}{lll}0 & 1 & 0\end{array}$ & $\mathrm{~A}^{\prime} \mathrm{B}^{\prime \prime} \mathrm{A}^{\prime} \mathrm{D}^{\prime}$ & 'D' \\
\hline & $\begin{array}{lll}0 & 1 & 1 / 2\end{array}$ & ${ }^{\prime} A^{\prime} B^{\prime \prime} C^{\prime \prime} C^{\prime}$ & $1 / 2$ \\
\hline & $\begin{array}{lll}0 & 1 & 1\end{array}$ & ${ }^{\prime} A^{\prime} B^{\prime \prime} B^{\prime \prime} B^{\prime}$ & 1 \\
\hline & $\begin{array}{lll}1 & 0 & 0\end{array}$ & ${ }^{\prime \prime} B^{\prime \prime} A^{\prime \prime} A^{\prime \prime} A^{\prime}$ & 0 \\
\hline & $10 \frac{1}{1}$ & ${ }^{\prime} B^{\prime \prime} A^{\prime \prime} C^{\prime \prime} C^{\prime}$ & $1 / 2$ \\
\hline & $\begin{array}{lll}1 & 0 & 1\end{array}$ & ${ }^{\prime} B^{\prime} A^{\prime \prime} B^{\prime \prime} D^{\prime}$ & 'D' \\
\hline & $11 / 20$ & ${ }^{\prime \prime} B^{\prime \prime} C^{\prime} A^{\prime \prime} C^{\prime}$ & $1 / 2$ \\
\hline & $11 / 21 / 2$ & ${ }^{\prime} B^{\prime \prime} C^{\prime \prime} C^{\prime \prime} C^{\prime}$ & $1 / 2$ \\
\hline & $11 / 21$ & ${ }^{\prime} B^{\prime \prime} C^{\prime \prime} B^{\prime \prime} B^{\prime}$ & 1 \\
\hline & 110 & ${ }^{\prime} B^{\prime \prime} B^{\prime \prime} A^{\prime \prime} B^{\prime}$ & 1 \\
\hline & $11 \frac{1}{1}$ & ${ }^{\prime} B^{\prime} B^{\prime \prime} C^{\prime} B^{\prime}$ & 1 \\
\hline & 111 & 'B'B'B'B'B' & 1 \\
\hline
\end{tabular}

Figure 8. Behaviour of the majority gate when constructed with ternary quantum-dot cells and the precondition that state ' $\mathrm{D}$ ' is allowed only for internal cells.

to the ternary AND logic function, and when its state is ' $\mathrm{B}$ ' the behaviour resembles the ternary OR logic function. In fact, in both cases there is only one erroneous output (i.e. $\operatorname{AND}(1,0)$ and $\operatorname{OR}(0,1))$. Furthermore, it can be noticed that there is an evident symmetry between the two cases, that in both cases the target cell assumes state ' $\mathrm{D}$ ', and that in both cases, when the inputs $X_{1}$ and $X_{2}$ are reversed, the target cell assumes the correct state: logic value 0 and 1 respectively. These properties aid our implementation substantially.

The solution can thus be based on a pair of majority gate structures, where both are subject to the same inputs $S, X_{1}$ and $X_{2}$, however, one of them has the inputs $X_{1}$ and $\mathrm{X}_{2}$ reversed. For most of the input combinations both structures will return the same output, which is a direct result of the symmetry of the AND and OR logic functions. The only cases when there are going to be differences are $\operatorname{AND}(1,0), \operatorname{AND}(0,1)$ and $\mathrm{OR}(0,1)$, OR $(1,0)$. For these cases, however, the following properties apply:

- the target cell of one of the two structures will assume state 'D', 
- the target cell of the other structure will assume the correct state and thus give the correct output.

In other words, this means that what is needed is a structure which will perform two tasks. In cases when two of its driver cells are in the same state the target cell must assume this state. In cases when one of these two driver cells is in state ' $D$ ' the target cell must assume the state of the other driver cells. A further study of the unconstrained behaviour of the majority gate constructed by using ternary quantum-dot cells presented in figure 7 reveals that such a structure is the majority gate itself, when the driver cell marked as $S$ is in state ' $C$ '. Indeed when the driver cells marked as $S, X_{1}$, and $X_{2}$ are in states ('C', X, X), ('C', X, 'D') or ('C', 'D', X) the target cell assumes state $\mathrm{X}$.

The final implementation of the ternary AND and OR logic functions as a hierarchy of three majority gate structures is presented in figure 9, whereas figure 10 presents

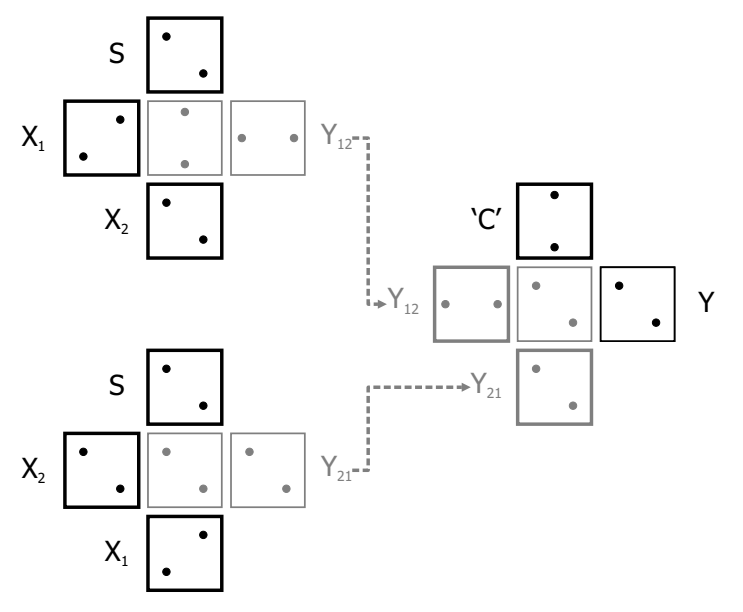

Figure 9. Implementation of the ternary AND and OR logic functions as a hierarchy of three majority gate structures.

\begin{tabular}{|c|c|c|c|c|}
\hline$S X_{1} X_{2}$ & $S X_{1} X_{2} Y_{12}$ & $S X_{2} X_{1} Y_{21}$ & ${ }^{\prime} C^{\prime} Y_{12} Y_{21} Y$ & $Y$ \\
\hline 000 & ${ }^{\prime} A^{\prime} A^{\prime \prime} A^{\prime \prime} A^{\prime}$ & ${ }^{\prime} A^{\prime} A^{\prime} A^{\prime} A^{\prime} A^{\prime}$ & ${ }^{\prime} C^{\prime \prime} A^{\prime} A^{\prime} A^{\prime}$ & $\overline{0}$ \\
\hline $\begin{array}{lll}0 & 0 & 1 / 2\end{array}$ & ${ }^{\prime} A^{\prime} A^{\prime \prime} C^{\prime} A^{\prime}$ & $A^{\prime} C^{\prime} C^{\prime} A^{\prime \prime} A^{\prime}$ & $C^{\prime} A^{\prime} A^{\prime} A^{\prime} A^{\prime}$ & 0 \\
\hline 001 & $\mathrm{~A}^{\prime} \mathrm{A}^{\prime} \mathrm{A}^{\prime \prime} \mathrm{B}^{\prime \prime} \mathrm{A}^{\prime}$ & ${ }^{\prime} A^{\prime} B^{\prime} A^{\prime} D^{\prime}$ & $C^{\prime} A^{\prime} A^{\prime \prime} D^{\prime} A^{\prime}$ & 0 \\
\hline $01 / 20$ & ${ }^{\prime} A^{\prime} C^{\prime} A^{\prime \prime} A^{\prime}$ & ${ }^{\prime} A^{\prime} A^{\prime \prime} C^{\prime} A^{\prime}$ & ${ }^{\prime} C^{\prime} A^{\prime} A^{\prime} A^{\prime}$ & 0 \\
\hline $0 \quad 1 / 21 / 2$ & $A^{\prime \prime} C^{\prime \prime} C^{\prime \prime} C^{\prime}$ & $A^{\prime \prime} C^{\prime \prime} C^{\prime \prime} C^{\prime}$ & ${ }^{\prime} C^{\prime \prime} C^{\prime \prime} C^{\prime \prime} C^{\prime}$ & $1 / 2$ \\
\hline $0 \quad 1 / 21$ & $A^{\prime \prime} C^{\prime \prime} B^{\prime \prime} C^{\prime}$ & $A^{\prime} B^{\prime \prime} C^{\prime \prime} C^{\prime}$ & ${ }^{\prime} C^{\prime \prime} C^{\prime \prime} C^{\prime \prime} C^{\prime}$ & $1 / 2$ \\
\hline $\begin{array}{lll}0 & 1 & 0\end{array}$ & ${ }^{\prime} A^{\prime} B^{\prime \prime} A^{\prime \prime} D^{\prime}$ & $A^{\prime} A^{\prime} B^{\prime \prime} A^{\prime}$ & ${ }^{\prime} C^{\prime \prime} D^{\prime \prime} A^{\prime} A^{\prime}$ & 0 \\
\hline $\begin{array}{lll}0 & 1 & 1 / 2\end{array}$ & ${ }^{\prime} A^{\prime} B^{\prime \prime} C^{\prime \prime} C^{\prime}$ & ${ }^{\prime} A^{\prime} C^{\prime \prime} B^{\prime \prime} C^{\prime}$ & ${ }^{\prime} C^{\prime \prime} C^{\prime \prime} C^{\prime \prime} C^{\prime}$ & $1 / 2$ \\
\hline $\begin{array}{lll}0 & 1 & 1\end{array}$ & $A^{\prime} B^{\prime \prime} B^{\prime \prime} B^{\prime}$ & $A^{\prime} B^{\prime} B^{\prime \prime} B^{\prime}$ & ${ }^{\prime} C^{\prime \prime} B^{\prime \prime} B^{\prime} B^{\prime}$ & 1 \\
\hline 100 & ${ }^{\prime} B^{\prime \prime} A^{\prime \prime} A^{\prime \prime} A^{\prime \prime}$ & ${ }^{\prime} B^{\prime \prime} A^{\prime \prime} A^{\prime \prime} A^{\prime \prime}$ & ${ }^{\prime} C^{\prime} A^{\prime} A^{\prime} A^{\prime} A^{\prime}$ & 0 \\
\hline $101 / 2$ & ${ }^{\prime} B^{\prime} A^{\prime \prime} C^{\prime \prime} C^{\prime}$ & ${ }^{\prime} B^{\prime \prime} C^{\prime \prime} A^{\prime \prime} C^{\prime}$ & ${ }^{\prime} C^{\prime \prime} C^{\prime \prime} C^{\prime \prime} C^{\prime}$ & $1 / 2$ \\
\hline $\begin{array}{lll}1 & 0 & 1\end{array}$ & ${ }^{\prime} B^{\prime \prime} A^{\prime \prime} B^{\prime \prime} D^{\prime}$ & ${ }^{\prime} B^{\prime \prime} B^{\prime \prime} A^{\prime \prime} B^{\prime}$ & $C^{\prime \prime} D^{\prime \prime} B^{\prime} B^{\prime}$ & 1 \\
\hline $11 / 20$ & ${ }^{\prime} B^{\prime \prime} C^{\prime \prime} A^{\prime \prime} C^{\prime}$ & ${ }^{\prime} B^{\prime \prime} A^{\prime \prime} C^{\prime \prime} C^{\prime}$ & ${ }^{\prime} C^{\prime \prime} C^{\prime \prime} C^{\prime \prime} C^{\prime}$ & $1 / 2$ \\
\hline $1 \frac{1 / 2}{1 / 2}$ & ${ }^{\prime} B^{\prime \prime} C^{\prime \prime} C^{\prime \prime} C^{\prime}$ & ${ }^{\prime} B^{\prime \prime} C^{\prime \prime} C^{\prime \prime} C^{\prime}$ & ${ }^{\prime} C^{\prime \prime} C^{\prime \prime} C^{\prime \prime} C^{\prime}$ & $1 / 2$ \\
\hline $11 / 21$ & ${ }^{\prime} B^{\prime \prime} C^{\prime \prime} B^{\prime \prime} B^{\prime}$ & ${ }^{\prime} B^{\prime} B^{\prime \prime} C^{\prime \prime} B^{\prime}$ & $C^{\prime} B^{\prime \prime} B^{\prime \prime} B^{\prime}$ & 1 \\
\hline $\begin{array}{lll}11 & 1\end{array}$ & ${ }^{\prime} B^{\prime} B^{\prime \prime} A^{\prime \prime} B^{\prime}$ & ${ }^{\prime} B^{\prime \prime} A^{\prime} B^{\prime \prime} D^{\prime}$ & ${ }^{\prime} C^{\prime \prime} B^{\prime \prime} D^{\prime \prime} B^{\prime}$ & 1 \\
\hline $11 \frac{1 / 2}{2}$ & ${ }^{\prime} B^{\prime \prime} B^{\prime \prime} C^{\prime \prime} B^{\prime}$ & ${ }^{\prime} B^{\prime \prime} C^{\prime} B^{\prime \prime} B^{\prime}$ & $C^{\prime \prime} B^{\prime \prime} B^{\prime \prime} B^{\prime}$ & 1 \\
\hline $\begin{array}{lll}11 & 1 & 1\end{array}$ & ${ }^{\prime} B^{\prime} B^{\prime \prime} B^{\prime \prime} B^{\prime}$ & ${ }^{\prime} B^{\prime} B^{\prime} B^{\prime \prime} B^{\prime}$ & ${ }^{\prime} C^{\prime} B^{\prime \prime} B^{\prime \prime} B^{\prime}$ & 1 \\
\hline
\end{tabular}

Figure 10. Behaviour of the structure implementing the ternary AND and OR logic functions.

the completed structure's behaviour. It is important to note that if the structure is 
constructed with binary quantum-dot cells and the input state ' $\mathrm{C}$ ' substituted with either logic value 0 or 1 the truth table of the binary AND and OR logic functions is obtained.

\section{Conclusion}

This article introduces the ternary quantum-dot cell, with the intention of enriching the processing capabilities of quantum-dot cellular automata (QCAs). By restricting to unclocked architectures and employing numerical simulations based on the semi-classical model [11] we have shown that electron tunnelling, associated with QCAs, can be used not only for binary but also for ternary processing. The analysis of ternary processing capabilities is based on the comparison with Łukasiewicz's ternary truth tables. From the computer science point of view the introduced cell and associated structures are capable of transferring, processing, and at least in theory also storing, crisp values in a ternary environment. In this view we find that, with the eventual increase in reliability, associated with technological progress, the ternary QCA structures are one of the possible candidates for the alternative processing platform of the future. Our current research is focused on ternary clocked architectures and ternary QCA structures capable of data storage.

\section{References}

[1] Steane A M and Rieffel E G 2000 IEEE Comput. 3338

[2] Phoenix C and Drexler E 2004 Nanotechnology 15869

[3] Lent C S, Tougaw P D, Porod W and Bernstein G H 1993 Nanotechnology 449

[4] Lent C S and Tougaw P D 1993 J. Appl. Phys. 746227

[5] Tougaw P D and Lent C S 1994 J. Appl. Phys. 751818

[6] Walus K, Dysart T J, Jullien G A and Budiman R A 2004 IEEE Trans. Nanotechnol. 326

[7] Hayes B 2001 Am. Sci. 89490

[8] Borkowski L (ed) 1970 Jan Lukasiewicz: Selected Works (Amsterdam: North-Holland Publishing Company)

[9] Tougaw P D and Lent C S 1996 J. Appl. Phys. 804722

[10] Snider G L, Orlov A O, Amlani I and Bernstein G H 1999 Japan. J. Appl. Phys. 387227

[11] Macucci M, Iannaccone G, Francaviglia S and Pellegrini B 2001 Int. J. Circ. Theor. Appl. 2937

[12] Lent C S and Tougaw P D 1997 Proc. IEEE 85541

[13] Niemier M T and Kogge P M 2004 Nano, Quantum and Molecular Computing ed S K Shukla and R I Bahar (Boston, MA: Kluwer Academic Publishers) p 267

[14] Kohavi Z 1978 Switching and finite automata theory (New York: McGraw-Hill Inc.)

[15] Walus K, Vetteth A, Jullien G A and Dimitrov V S 2003 Proc. Nanotech 2160

[16] Lebar Bajec I and Mraz M 2005 From Cellular Automata to Wetware ed C Teucher and A Adamatzky (Beckington: Luniver Press) p 95 